\title{
The Purification and Composition Analysis of Pentosans from Wheat Bran
}

\author{
Baiying Cao ${ }^{1, a}$ Baolan Bai, ${ }^{2, b}$ Xiaoqiu Liu ${ }^{3, c}$ \\ 123 Jilin Engineering Normal University, Changchun, Jilin, China, 130052 \\ aemail, ${ }^{\text {bemail, }}{ }^{\mathrm{c} e m a i l,}$
}

Keywords: Purification, Composition Analysis, Pentosans, Wheat Bran

\begin{abstract}
Our country is the largest producer of wheat and in the processing of wheat, the yield of bran is nearly $20 \%$ of wheat processing capacity, the only by-product of wheat processing to produce each year - the bran as high as more than 20 million t, which is a big resources. The main contents of this part of the study are prepared from bran pentosan. The pentosan is prepared and purified grade, and the composition of each component is analyzed for future pentosan structural analysis, functional and structural test the relationship between the functions of the foundation.
\end{abstract}

\section{Introduction}

Pentosan is as a high molecular weight structural polysaccharide, a number of important physical and chemical properties, giving pentosan some unique features and uses, water characteristics, high viscosity characteristics, oxidation and other properties such as absorbent gelling hold. Pentosan is a non-starch polysaccharides, mainly of pentose sugars (arabinose and xylose), with widely present in the outer layer of the grain of wheat, rye, barley, etc., less inner endosperm content. Amyl poly sugar water has a high water retention characteristics, high viscosity characteristics, oxidation and the formation of crosslinked gel, emulsion stability and so on, the quality of dough properties and flour products have an important impact. Research on pentosan mainly from cereals cortex pentosan extracted, which was developed as thickeners, humectants, emulsion stabilizers used in food industry and cosmetics industries. In addition, pentosan also has colon cancer and breast cancer prevention and control, lower blood sugar and weight loss, etc. effect, can be added as a factor to functional health foods. In this paper, wheat bran, water as the extraction solvent prepared WSP, respectively ethanol precipitation, ion exchange chromatography and gel filtration chromatography methods WSP prepared crude was purified grade, and its composition was analyzed.

\section{The Biological Value of Pentosan}

In other countries, through long-term research on wheat pentosan nature, the results showed that: pentosan have a major impact on the quality of baked bread, flour add pentosan can significantly improve the quality of wheat bread baked in pentosan. mainly in the outer portion of wheat that is a byproduct of wheat processing - bran, pentosan content accounted for about $20 \%$ of Thus, bran, pentosan preparation, which was developed as bread additives, and has good development prospects. in addition, pentosan has properties of high viscosity, gelation, etc. oxidation, can also be used as a thickener and a humectant used in the food industry. in addition, pentosan as a functional polysaccharide, but also has very important physiological functions, according to research data shows that pentosan has a slimming, laxative, lipid-lowering, antioxidant and some other important physiological functions.

Our country is the largest producer of wheat in the processing of wheat, a yield of nearly $20 \%$ wheat bran processing capacity, the only by-product of wheat processing to produce each year - the bran as high as more than 20 million t, which is a big resources. at present in our country, mainly wheat bran for direct use as animal feed, and other fermentation medium, low prices for many large-scale production and processing enterprises, flour, bran can be achieved if the proliferation and effective use of the It has a very important economic and social benefits. 


\section{The Related Research on Pentosan}

Pentosan as an oligosaccharide, widely present in the outer layer of the grain of wheat, rye, barley, etc., less inner endosperm content. Gupi in wheat pentosan content of about 20\%, and therefore the drum skin was prepared from wheat pentosan and has good development prospects. It mainly are polysaccharides pentose, xylose, arabinose, small amounts of galactose, mannose, glucose and the like thereof. Numerous studies indicate that pentosan has high water absorption properties of water retention, high viscosity characteristics and oxidation crosslinked to form a gel, emulsion stabilizing role, quality Dough Properties and flour products have an important impact. The nature of high viscosity, gelation, etc. oxidation, can also be used as a thickener and a humectant used in the food industry. Additionally, pentosan polysaccharide as an active laxative, lowering blood pressure and anti-tumor effects, particularly for the prevention and control of cancer has a significant effect. Pentosan based on the solubility can be divided into soluble and insoluble pentosan. In summary, the preparation of soluble pentosan first wheat in the number of skin-soluble substances and water-insoluble material separated and then dissolved in water, starch, protein, dextran, galactose Arabia and other impurities removed [period. When preparing the insoluble pentosans, the first drum skin was degreased wheat, starch and protein to give the water-insoluble substance, and then the delignification, and then with a saturated calcium hydroxide solution was extracted with different concentration of the final ethanol precipitation and you can get a water-insoluble pentosan. Zheng Xueling drum skin such as the use of wheat as raw material, washed with water and a base for the preparation of an aqueous extraction solvent water-insoluble pentosan, column chromatography, and then the two methods was precipitated with ethanol and ion exchange of pentosan prepared grading purified, fractionated components mainly composed of monosaccharides arabinose and xylose.

\section{Preparation of Pentosan with Wheat Bran}

Pentosan wheat in major non-starch polysaccharides, one of the main components of dietary fiber cereal composition, it is mainly by the polysaccharide pentose, xylose, arabinose, a small amount of galactose, mannose, glucose and consisting. Numerous studies indicate that pentosan dough properties have significant effects and the impact of the baking quality bread wheat pentosan mainly found in wheat bran, wheat bran contains about $20 \%$ pentosan. So be prepared with wheat bran pentosan as raw material, which was developed as bread additives, and has good development prospects, in addition, pentosan oxide gel having a high viscosity and other properties, but also as a thickener and moisturizing agents used in the food industry.

\section{Preparation of Water-Soluble Pentosans}

Wheat bran with mill after grinding through a 40 mesh sieve, sieve material by $\mathrm{W}$ (bran): $\mathrm{V}$ (volume fraction of $80 \%$ ethanol) $=1 \mathrm{~g}: 5 \mathrm{ml}$ of proportion, $70{ }^{\circ} \mathrm{C}$ under reflux for $2 \mathrm{~h}$, centrifuged supernatant; insolubles mass ratio of $1: 10$ by adding distilled water, and adding a certain amount of pentosan enzyme, $60{ }^{\circ} \mathrm{C}$ extraction $2 \mathrm{~h}$; the supernatant was centrifuged, the supernatant was heated to $95{ }^{\circ} \mathrm{C}$, adding the right amount of heat à- amylase, incubated $90 \mathrm{~min}$, and continue to oscillate, then cooled to $60{ }^{\circ} \mathrm{C}$, adding an appropriate amount of glucoamylase, heat $1 \mathrm{~h}$; then treated with a neutral protease to decompose protein contained therein, is heated to $100{ }^{\circ} \mathrm{C}$, and held for $20 \mathrm{~min}$ processed to inactivate enzymes; the supernatant was centrifuged, the supernatant was concentrated to a certain volume, and then 4 volumes of ethanol was added, still at $4{ }^{\circ} \mathrm{C}$ overnight; the precipitate was collected by centrifugation, the precipitated fraction plus a certain amount dissolved in water, concentrated to remove residual ethanol, to give a water soluble pentosan crude product (C-WSP).

\section{DEAE-Cellulose Column Chromatography Grade}

The use of DEAE-cellulose column using $\mathrm{H} 2 \mathrm{O}$ and different ionic strength $\mathrm{NaCl}$ elution each tube to collect $5 \mathrm{ml}$, with phenol - sulfuric acid method in the determination of the total sugar content of 
$480 \mathrm{~nm}$ as follows:

WSP or AEP solution was heated to $92{ }^{\circ} \mathrm{C}$, incubated $30 \mathrm{~min}$, cooled TCA was added to a concentration of about $7 \%, 4{ }^{\circ} \mathrm{C}$ overnight resting, centrifugation (10 000g10 min), the supernatant was dialyzed $72 \mathrm{~h}$, over DEAE-cellulose column, and $\mathrm{H} 2 \mathrm{O}$ respectively $0.1,0.5 \mathrm{~mol} / \mathrm{L} \mathrm{NaCl}$ for elution, detection of total sugar, collected by different components.

\section{Component Analysis of WSP and AEP Composition Graded}

By gas chromatography (GC method) with a strong acid WSP, AEP hydrolyzed to monosaccharides, monosaccharide then acetylated, converted to the respective sugar alcohol, and analyzed by GC.

Sample treatment: Weigh $0.5 \mathrm{~g}$ sample in hydrolysis tube, add $1 \mathrm{~mol} / \mathrm{L}$ sulfuric acid for about 5 $\mathrm{ml}$, hydrolysis tube was sealed at 100e hydrolysis $4 \mathrm{~h}$, with barium carbonate, filtered, and the filtrate was dried under vacuum placed $45^{\circ} \mathrm{C}$. was added $0.5 \mathrm{ML}$ of pyridine was dissolved monosaccharides, then add 10mg of hydroxylamine hydrochloride and $6 \sim 8 \mathrm{mg}$ of inositol, in $90^{\circ} \mathrm{C}$ bath for $0.5 \mathrm{~h}$, removed after cooling, was added $0.5 \mathrm{ml}$ of acetic anhydride at $90^{\circ} \mathrm{C}$ bath for $30 \mathrm{~min}$ (reaction prevent water from entering the process). after cooling, the same sample analyzed by GC monosaccharide standard approach .GC conditions: using GC-14A gas chromatograph, CR-4A integrator, OV170 quartz glass capillary column, FID detector, N2 volumetric flow rate of $2 \mathrm{ml} /$ min, $\mathrm{H} 2$ volume flow rate of $40 \mathrm{ml} / \mathrm{min}$, air volume flow rate of $550 \mathrm{ml} / \mathrm{min}$, the temperature of the gasification chamber $270^{\circ} \mathrm{C}, 250^{\circ} \mathrm{C}$ temperature detector temperature programming mode: to $3^{\circ} \mathrm{C} / \mathrm{min}$ of rate rise by the $190^{\circ} \mathrm{C} 240^{\circ} \mathrm{C}$, the injection volume was $1 \mathrm{~L}$.

\section{Preparation and Chemical Composition of Pentosan Crude Extract}

Wheat Bran pentosan according to their solubility can be divided into water-soluble and water-insoluble pentosan pentosan, were extracted with water and lye. Lye OH- can break pentosan and lignin in cell walls, non-covalent interactions between cellulose and adjacent pentosan ferulic acid covalently crosslinked to promote cell wall expansion financial structure loose, the insoluble polymer to become water-soluble polymer, and thus in favor of free pentosan, pentosan improve the extraction rate. Commonly used bases are $\mathrm{NaOH}, \mathrm{KOH}, \mathrm{Ba}(\mathrm{OH}) 2$, $\mathrm{Ca}(\mathrm{OH}) 2$ and the like.

Using $\mathrm{NaOH}$ as an extraction medium to extract not only water insoluble pentosans, but may be

$\beta$ - glucan and hemicellulose .Therefore, the polysaccharide is prepared containing a multi-component mixture. Saturated $\mathrm{Ba}(\mathrm{OH}) 2$ solution is the most effective medium pentosan isolated from the cell walls of many cereals. In addition to the action of the $\mathrm{OH}-, \mathrm{Ba} 2+$ can specifically and arabinose (Ara) and xylose (Xyl) and other pentose combine to make a separate dissolution of pentosan to obtain purer pentosan.

\section{Yield and Composition of the Soluble Pentosan and the Water-Insoluble Pentosan}

Using water and an aqueous base as solvent extraction of water-insoluble pentosan and pentosan two extracts yield and composition shown in Table 1.

Table 1 yield and composition of preparation two components

\begin{tabular}{lcccc}
\hline Grade & Yield/\% & Protein Content $/ \%$ & Total sugar $/ \%$ & Pentosan $/ \%$ \\
\hline WSP & 7.7 & 14.3 & 67.4 & 56.5 \\
AEP & 11.9 & 16.5 & 60.3 & 60.7 \\
\hline
\end{tabular}

As it can be seen from Table 1, the water-soluble and water-insoluble pentosan pentosan crude, in addition to containing about $60 \%$ pentosan, but also contains a lot of protein, because this experiment did not specifically to remove protein, raw bran also contains large amounts of protein (15\%), of which a large part of the protein is water soluble and alkali-soluble, so that water and base as solvent extraction pentosans, a large part of the protein is also extracted. 


\section{Yield and Composition Analysis of Pentosan Solvent Precipitation Method}

Water-soluble and water-insoluble pentosan water and a base for the preparation of a solvent containing more components of crude, in order to study the relationship between pentosan composition, structure and between the structure and function of the need for prepared pentosan purified grade. It is fractionated by solvent precipitation yield components of the composition shown in table 2

Table 2 Yield solvent precipitation grade components and composition\%

\begin{tabular}{|c|c|c|c|c|c|c|c|c|}
\hline \multirow[t]{2}{*}{ Grade } & \multirow[t]{2}{*}{ Yield $^{\mathrm{a}}$} & \multirow[t]{2}{*}{ Protein } & \multirow[t]{2}{*}{ Total } & \multirow{2}{*}{$\begin{array}{l}\text { Major } \\
\text { Ara }^{\mathrm{C}}\end{array}$} & \multicolumn{2}{|c|}{ component } & \multicolumn{2}{|c|}{ monosaccharides $^{b}$} \\
\hline & & & & & $\mathrm{Xyl}^{\mathrm{d}}$ & $\mathrm{Glc}^{\mathrm{c}}$ & $\mathrm{Gal}^{\mathrm{f}}$ & Ara/Xyl ${ }^{g}$ \\
\hline WSP1 & 0.63 & 22.01 & 68.62 & 15.1 & 68.2 & 4.5 & 0.4 & 0.22 \\
\hline WSP2 & 1.53 & 9.15 & 82.50 & 18.2 & 61.4 & 13.5 & 0.8 & 0.30 \\
\hline WSP3 & 2.20 & 6.41 & 87.42 & 40.9 & 48.6 & 2.1 & 7.1 & 0.84 \\
\hline WSP4 & 1.85 & 12.81 & 80.59 & 17.0 & 14.3 & 65.8 & 0.3 & 1.19 \\
\hline AEP1 & 1.84 & 37.21 & 57.22 & 17.4 & 66.4 & 7.5 & 0.4 & 0.27 \\
\hline AEP2 & 2.93 & 7.44 & 80.93 & 23.2 & 59.5 & 3.3 & 0.7 & 0.39 \\
\hline AEP3 & 3.57 & 4.55 & 89.37 & 45.5 & 42.7 & 1.1 & 2.4 & 1.07 \\
\hline AEP4 & 3.07 & 9.56 & 85.21 & 16.0 & 12.7 & 61.8 & 0.3 & 1.26 \\
\hline
\end{tabular}

$\mathrm{A}$ is the yield of each component expressed as a percentage of an amount of each raw bran components; b each represents the percent of the composition of a monosaccharide; C Representative arabinose; D-xylose Representative; E representative of glucose; F. Representative galactose ; the ratio of $g$ on behalf of arabinose and xylose

\section{Conclusion}

Precipitation with a solvent and column chromatography on a good bran soluble and water-insoluble pentosan crude extract was classified as grade components have different protein contents and different monosaccharide composition. As the precipitating solvent the concentration increases, the classification of pentosan fraction containing higher Ara / Xyl ratio. by DEAE composition analysis and the average molecular weight cellulose classification of the components measured and found to have a higher Ara / Xyl ratio group points having a high average molecular weight.

\section{Acknowledgements}

Fund Project: Jilin Province Department of Education "The Twelfth Five-Year Guideline" scientific and technological research projects, Kyrgyzstan UNESCO co-word [2014] No. 564

\section{References}

[1] Jia Xinzhang, Li Jingyuan. Cereal Chem, Vol. 6 (2014) No 53, p.25-26

[2] Peng Sue, Wang Yunhui, Wang Qunyong. Carbohydrate Polymers, Vol. 12 (2015) No 27, p.74-76

[3] Qian Xiyuan, Jing Jianfen, Hou XuSiem. Journal of Cereal Science, Vol. 30 (2014) No 19, p.144-145

[4] Wang Kuailiang. Cereal Chem, Vol. 29 (2011) No 27, p.21-23

[5] Zhang Gongxu, Sun Jing. Feed Industry, Vol. 8 (2013) No 27, p.57-60 